\title{
Conditions aux limites liées à la réflexion de la houle et distribution des énergies dans un bassin portuaire.
}

\author{
Martin SANCHEZ \\ Maitre de conférences, LMG, Université de Nantes
}

\begin{abstract}
Résumé
La théorie linéaire de propagation de la houle monochromatique considère l'existence d'une fonction potentielle réduite bidimensionnelle complexe caractérisant l'amplitude de la houle et sa phase dans chaque point du domaine examiné. Dans ce domaine, la fonction potentielle réduite est régie par l'équation de Helmholtz avec des conditions aux limites appropriées, tenant compte de la houle incidente et des éventuelles réflexions au niveau des parois délimitant le domaine étudié.

Cette étude concerne la simulation de la propagation de la houle dans un bassin rectangulaire ; l'équation de Helmholtz est résolue en utilisant la méthode implicite de différences finies avec un schéma centré aux noeuds à l'intérieur du domaine et un schéma décentré sur les frontières. Le maillage utilisé comporte 36 noeuds par longueur d'onde ; ce qui conduit à un total de 27577 noeuds dans cette étude.

L'éventuelle réflexion nulle ou partielle des parois latérales est prise en compte grâce à une formulation mathématique originale considérant le coefficient de réflexion de ces frontières.

Enfin, on étudie les répartition de l'énergie potentielle, de l'énergie cinétique et de l'énergie totale dans le bassin. De même, on étudie le flux d'énergie transmise et la répartition des phases. Cette façon peu courante de présenter les résultats permet de mieux comprendre la circulation d'énergie engendrée par la houle incidente.
\end{abstract}

\section{Introduction}

Un modèle permettant la simulation de la houle monochromatique dans un bassin portuaire de forme arbitraire a été développé par Berkhoff [1]. Selon cette approche, les vitesses dérivent d'un potentiel complexe $\Phi$ régi par l'équation de Laplace. D'une part, la forme générale de ce potentiel lui permet de satisfaire les conditions aux limites liées à limperméabilité du fond marin et à la pression constante sur la surface libre de l'eau. D'autre part, les conditions aux limites du domaine étudié en communication avec la mer et celles liées aux parois (lesquelles sont caractérisées par un coefficient de réflexion réel $\mathrm{K}_{\digamma}$ ) doivent être déterminées cas par cas. Selon Berkhoff la forme générale de ce potentiel est la suivante :

$\Phi=-\frac{i g a_{0}}{\omega} \phi(x, y) \frac{\cosh [k(z+d)]}{\cosh [k d]} \exp (-i \omega t)$ 
où $\phi(x, y)$ est une fonction potentielle réduite bidimensionnelle complexe restant à déterminer, $g$ l'accélération de la pesanteur, $a_{0}$ l'amplitude de l'onde incidente de référence, d la profondeur, $\mathrm{i}=(-1)^{1 / 2}$, $\mathrm{x}$ et $\mathrm{y}$ les coordonnées horizontales, $\mathrm{z}$ la coordonnée verticale, $t$ le temps, $k$ le nombre d'onde et $\omega$ la fréquence angulaire $; k$ et $\omega$ sont reliés par la relation de dispersion ci-dessous :

$\omega^{2}=\mathrm{gk} \tanh [\mathrm{kd}]$

Lorsque d est constant la fonction potentielle réduite est gouvernée par l'équation de Heimholtz:

$\frac{\partial^{2} \phi}{\partial x^{2}}+\frac{\partial^{2} \phi}{\partial y^{2}}+k^{2} \phi=0$

La solution de cette équation doit satisfaire à des conditions aux limites appropriées associées à chaque site étudié.

\section{Conditions aux limites à la frontière entre le domaine étudié et la mer}

Si la frontière entre le domaine étudié et la mer est dềsignée par $\Gamma$, et la fonction potentielle réduite caractérisant l'onde progressive incidente de référence par $\phi_{\mathrm{w}}$, la fonction potentielle réduite $\phi$ sur cette frontière est donnée par :

$\phi=\phi_{\mathrm{w}}+\phi_{\mathrm{pe}} \quad$, avec : $\phi_{\mathrm{w}}=\exp \left[\mathrm{ik}\left(\mathrm{x} \cos \theta_{\mathrm{w}}+\mathrm{y} \sin \theta_{\mathrm{w}}\right)\right] \quad \underline{\operatorname{sur} \Gamma}$

où $\phi_{\mathrm{pe}}$ est la fonction potentielle réduite liée à l'onde perturbée qui se propage vers l'extérieur du domaine étudié et $\theta_{\mathrm{w}}$ l'angle entre la direction d'incidence de la houle de référence et l'axe $\mathrm{Ox}$. La condition de continuité de la vitesse normale à cette frontière impose alors la condition suivante :

$\frac{\partial \phi}{\partial \mathrm{n}}=\frac{\partial \phi_{\mathrm{w}}}{\partial \mathrm{n}}+\frac{\partial \phi_{\mathrm{pe}}}{\partial \mathrm{n}} \quad$, avec: $\frac{\partial \phi_{\mathrm{pe}}}{\partial \mathrm{n}}=-\mathrm{ik} \phi_{\mathrm{pe}} \cos \gamma_{\mathrm{pe}} \quad \underline{\text { sur } \Gamma}$

où n est la normale à la frontière entre le domaine étudié et la mer ouverte, positive vers l'intérieur du domaine étudié, et $\gamma_{p e}$ l'angle entre la direction d'incidence de l'onde perturbée se propageant vers l'extérieur du domaine étudié et $n$.

\section{Conditions aux limites liées aux parois délimitant le bassin}

Lorsque les parois sont verticales et parfaitement réfléchissantes, la condition de frontière acceptée couramment est la suivante :

$\frac{\partial \phi}{\partial n}=0$ 
Dans le cas d'une frontière partiellement réfléchissante, les approches diffèrent selon les auteurs ; une condition proposée par Berkhoff [1] est :

$\frac{\partial \phi}{\partial \mathrm{n}}+\left(\alpha_{1}+\mathrm{i} \alpha_{2}\right) \mathrm{k} \phi=0$

où $\left(\alpha_{1}+\mathrm{i} \alpha_{2}\right)$ est un coefficient de transmission complexe. Isaacson et $\mathrm{Qu}$ [2] présentent des expressions de $\alpha_{1}$ et de $\alpha_{2}$ déduites de l'étude d'une onde progressive abordant une paroi partiellement réfléchissante, laquelle est caractérisée par son coefficient de réflexion $\mathrm{K}_{\mathrm{I}}$ et par son déphasage de réflexion $\beta$. La valeur de $\beta$ dépend fortement du positionnement physique de la paroi dans le modèle par rapport à celui du prototype. Avec un positionnement approprié, difficile à déterminer certes, $\beta=0$. Dans cette étude, on considère cette dernière égalité comme hypothèse, ce qui conduit aux expressions simplifiées qui suivent :

$\alpha_{1}=0 \quad$ et $\quad \alpha_{2}=\frac{1-K_{r}}{1+K_{r}} \cos \gamma$

où $\gamma$ est l'angle entre la direction d'incidence de la houle et $\mathrm{n}$. La difficulté pour appliquer cette expression est double. D'un côté, la direction d'incidence de la houle sur une paroi n'est pas connue à priori. D'un autre côté, lorsque l'agitation est stationnaire et/ou tridimensionnelle, la notion de direction de propagation de la houle perd toute signification. On est alors obligé de donner une définition pour la direction de la propagation de la houle qui soit la plus réaliste possible.

$\mathrm{Si}$ l'on considère une onde progressive cylindrique abordant obliquement une paroi localisée en $x=0$, laquelle est caractérisée par son coefficient de réflexion $\bar{K}_{\mathrm{T}}$, la fonction potentielle réduite du champ de houle résultant est donnée par :

$\phi=A\left(\exp [i k(x \cos \gamma+y \sin \gamma)]+K_{r} \exp [-i k(x \cos \gamma-y \sin \gamma)]\right\}$

où A est le module de la fonction potentielle réduite correspondant à l'onde incidente sur la paroi. En dérivant l'équation (9) par rapport à n, on obtient :

$\frac{\partial \phi}{\partial \mathrm{n}}=\mathrm{i} \frac{\partial \psi}{\partial \mathrm{n}} \phi \quad$, avec $: \quad \frac{\partial \psi}{\partial \mathrm{n}}=-\frac{1-\mathrm{K}_{\mathrm{r}}}{1+\mathrm{K}_{\mathrm{r}}} \mathrm{k} \cos \gamma$

Afin d'approcher cette dernière équation, une démarche originale a été suivie. Une première estimation de $\phi$ est obtenue en prenant $\gamma=0^{\circ}$ sur toutes les parois. Pour la deuxième estimation, on calcule les conditions aux limites en utilisant l'expression (11) ci-dessous (développée dans cette étude) :

$\frac{\partial \phi}{\partial \mathrm{n}}=\mathrm{i} \frac{\partial \psi}{\partial \mathrm{n}} \phi \quad$, avec $: \quad 0 \leq-\frac{\partial \psi}{\partial \mathrm{n}} \leq \frac{1-K_{\mathrm{T}}}{1+\mathrm{K}_{\mathrm{r}}} \mathrm{k}$

où $\psi$ est la phase de la fonction potentielle réduite $(\phi=A \exp (i \psi))$. Par la suite, on répète ce processus jusqu'à l'invariabilité des résultats obtenus. D'une façon pratique, trois itérations suffisent habituellement. 


\section{Crandeurs adimemsionnelles caractéristiques de la houle}

\subsection{Energie potentielle}

Habituellement l'agitation en chaque point d'un bassin est caractérisée par le rapport de l'amplitude locale de la houle sur l'amplitude de la houle incidente de référence. Afin de pouvoir étudier par la suite la répartition de l'énergie totale, l'agitation sera caractérisée, dans cette étude, par l'énergie potentielle (moyenne sur un cycle de marée) par unité de surface horizontale, laquelle est donnée par:

$\mathrm{Ep}=\frac{1}{4} \rho \mathrm{g} \mathrm{a}_{0}^{2}\left(\mathrm{f}^{2}+\mathrm{g}^{2}\right)$

où $f$ et $g$ sont respectivement les parties réelle et imaginaire de la fonction potentielle réduite : $\phi=f+i \mathrm{~g}$. Alors, une énergie potentielle adimensionnelle Ep* peut être définie comme suit :

$E p^{*}=\frac{4 E p}{\rho g a_{0}^{2}}=f^{2}+g^{2}$

Le terme Ep* est le carré du rapport utilisé habituellement pour caractériser l'agitation; pour la houle incidente $\mathrm{Ep}^{*}=1$.

\section{2. Énergie cinétique}

L'énergie cinétique due au mouvement horizontal Ech par unité de surface est donnée par l'expression suivante :

$E c h=\frac{1}{4} \rho g a_{0}^{2} \frac{C_{E}}{C}\left[\left(\frac{\partial f}{\partial k x}\right)^{2}+\left(\frac{\partial g}{\partial k x}\right)^{2}+\left(\frac{\partial f}{\partial k y}\right)^{2}+\left(\frac{\partial g}{\partial k y}\right)^{2}\right]$

où $C_{E}$ est la vitesse de propagation de l'énergie, égale à la célérité de groupe, et $C$ la célérité d'onde. La répartition de l'énergie cinétique est liée à la profondeur relative ; dans cette étude on examine exclusivement le cas des fäibles profondeurs $\left(C_{E}=C\right)$. Dans ce cas, l'énergie cinétique liée au mouvement vertical peut être négligée et l'on peut définir une énergie cinétique adimensionnelle totale Ec* comme suit :

$E c^{*}=\frac{4 E c h}{\rho g a_{0}^{2}}=\left[\left(\frac{\partial f}{\partial k x}\right)^{2}+\left(\frac{\partial g}{\partial k x}\right)^{2}+\left(\frac{\partial f}{\partial k y}\right)^{2}+\left(\frac{\partial g}{\partial k y}\right)^{2}\right]$

Pour la houle incidente de référence $\mathrm{Ec} *=1$.

\section{3. Énergie totale}

Cette énergie est la somme des deux précédentes ; adimensionnellement on écrira :

$\mathrm{E}^{*}=\mathrm{Ep}^{*}+\mathrm{Ec}^{*}$

Pour la houle progressive incidente de référence $E^{*}=2$. 


\subsection{Phase de la houle}

La phase de la houle $\psi$ ou phase de la fonction potentielle réduite, peut être calculée grâce à l'expression suivante :

$\psi=\arg (f+i g)$

Pour $\mathrm{f}=\mathrm{g}=0$, la phase est indéfinie ; ceci est la cas dans les points amphidromiques où l'amplitude est nulle.

\section{5. Énergie transmise ou flux d'énergie}

L'énergie transmise adimensionnelle $\mathrm{E}^{*}$ sera définie par l'expression suivante :

$E t^{*}=\frac{2 E t}{\rho g a_{0}^{2} C_{E}}=\left[f \frac{\partial g}{\partial k x}-g \frac{\partial f}{\partial k x}+f \frac{\partial g}{\partial k y}-g \frac{\partial f}{\partial k y}\right]$

où Et est l'énergie transmise par la houle. Le calcul de l'énergie transmise par la houle incidente conduit à $\mathrm{Et}^{*}=1$. Il faut noter que l'énergie transmise est représentée par un vecteur ayant une composante selon $\mathrm{x}$ et une composante selon $\mathrm{y}$.

\section{Configuration du bassin étudié}

Il s'agit d'un bassin rectangulaire à profondeur constante ayant été étudié par plusieurs auteurs $[2,3]$. Cette configuration simple est représentée sur la figure 1 . On considérera dans tous les cas une incidence de la houle suivant l'axe de symétrie du bassin.

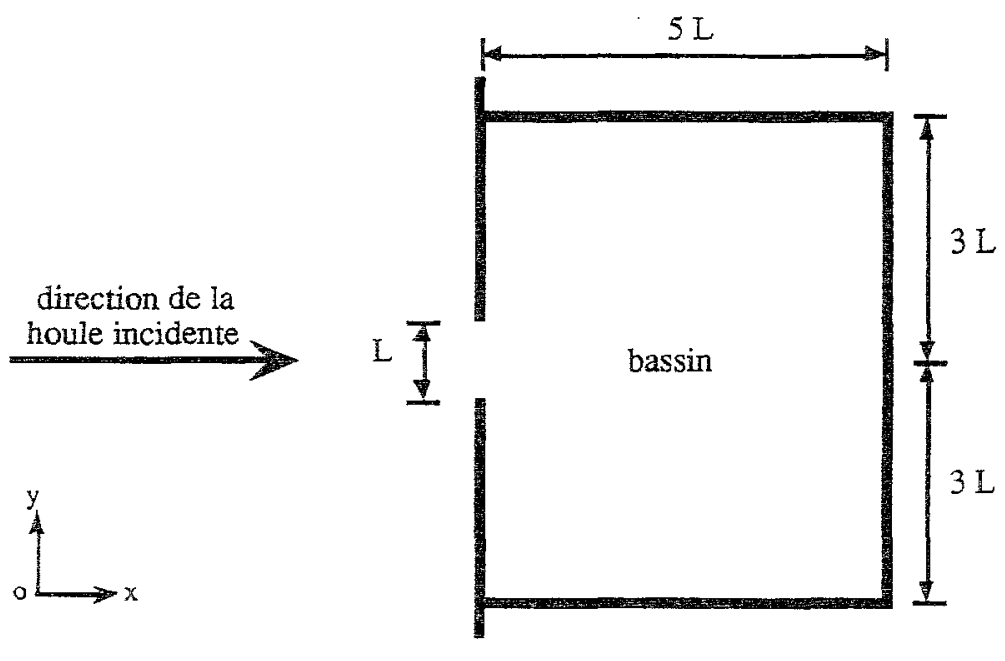

I: longueur d'onde

Fig. 1. Configuration du bassin étudié. 


\section{Agitation dans un assin avec des parois complètement absorbantes}

La figure 2 montre la distribution de l'énergie potentielle adimensionnelle Ep* évaluée après deux itérations de caicul. La solution analytique de Blue et Johnson [4] et les mesures expérimentales de Pos et Kilner [3] sont représentées sur cette même figure. On constate que les résultats numériques s'approchent de la solution analytique ; cependant, ils montrent un écart appréciable vis-à-vis des résultats expérimentaux, lequel peut être expliqué par les difficultés inhérentes aux essais en laboratoire lorsque l'on désire reprođuire une absorption totale de l'énergie par les parois.

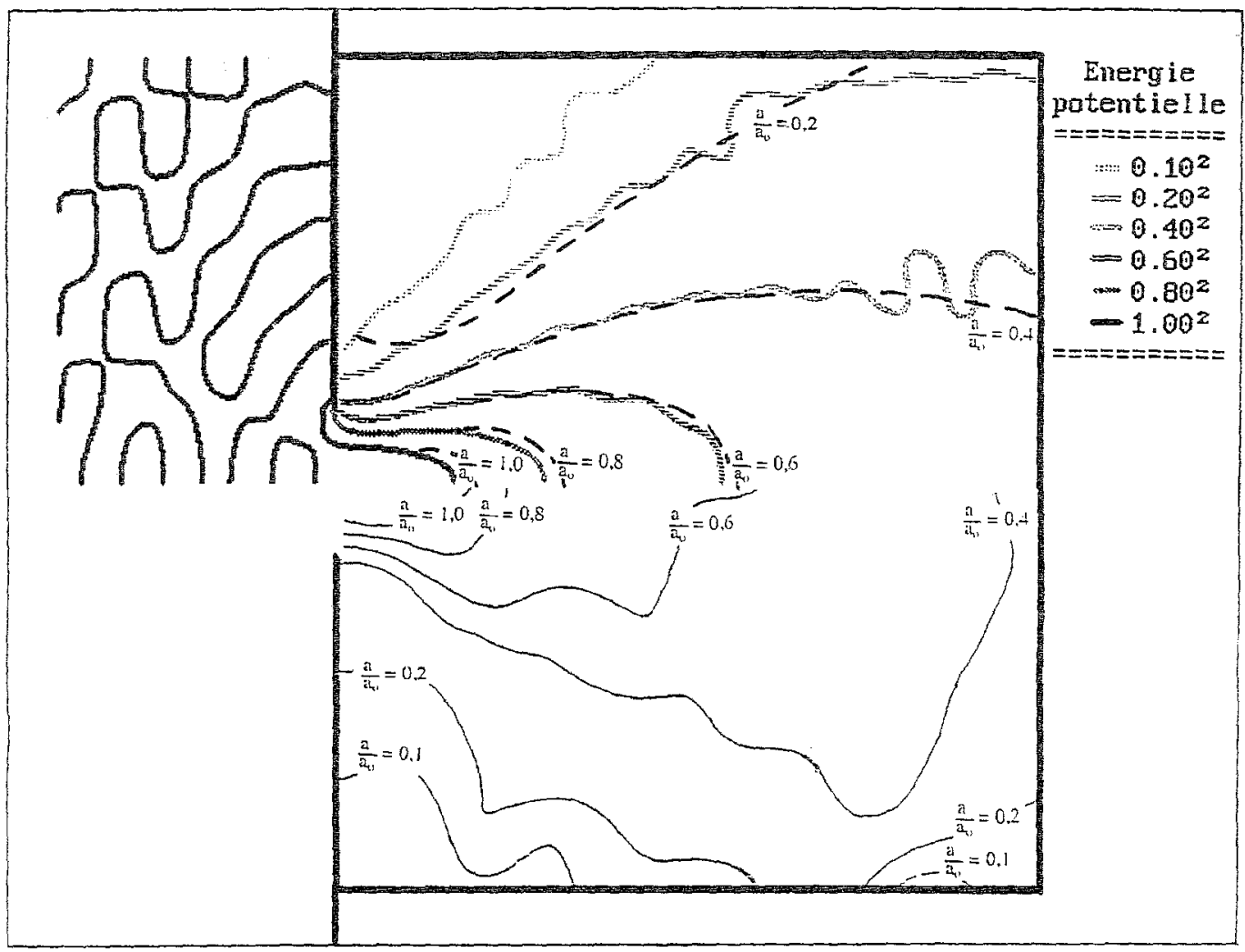

Fig.2.- Agitation dans un bassin entouré de frontières complètement absorbantes.

\section{Moitié supérieure du bassin}

- En traits continus : distribution de Ep* -l'amplitude locale de la houle sur l'amplitude de la houle incidente vaut $E p^{* 1 / 2}$ - (d'après cette étude).

- En traits discontinus : ampiitude locale de la houle sur amplitude de la houle incidente de référence (d'après la solution analytique de Blue et Johnson [4]).

Moitié inférieure du bassin

- Amplitude locale de la houle sur amplitude de la houle incidente de référence (d'après les mesures expérimentales de Pos et Kilner [3]). 


\section{Agitation dans un bassin aux parois parfaitement réfléchissantes}

Dans ce cas, les conditions aux limites liées aux parois sont bien connues (équation (6)) ; la solution numérique peut être obtenue directement en une seule itération. L'analyse des résultats obtenus donne des renseignements intéressants afin de mieux comprendre le phénomène de propagation de la houle dans un bassin. Malheureusement, il n'existe pas de solution analytique ou expérimentale de ce problème permettant la validation du modèle utilisé.

\subsection{Champs des énergies potentielle, cinétique et totale.}

La figure 3 montre la distribution de l'énergie potentielle adimensionnelle Ep* dans un bassin entouré de parois parfaitement réfléchissantes. On y observe la formation d'un clapotis avec des ventres d'oscillation le long de la paroi du fond du bassin et sur des lignes parallèles à cette paroi, séparées entre elles d'une demi-longueur d'onde, les noeuds d'oscillation se localisant à mi-distance entre deux ventres. On constate également un phénomène de résonance à l'intérieur du bassin conduisant à des amplitudes jusqu'à trois fois supérieures à celle de la houle incidente, alors qu'à l'extérieur du bassin les amplitudes maximales n'atteignent que deux fois cette amplitude.

La figure 4 montre la distribution de $\mathrm{Ec}^{*}$ dans le même cas que précédemment. Si l'on compare avec la figure 3 , on observe que les maximums d'énergie cinétique sont localisés sur les noeuds d'oscillation (là où l'énergie potentielle présente un minimum) et les minimum sur les ventres (là où l'énergie potentielle présente un maximum). De même, on observe que les valeurs maximales de Ep* et de Ec* sont du même ordre de grandeur.

La figure 5 montre la distribution de l'énergie totale adimensionnelle $\mathrm{E}^{*}$. On constate que dans le domaine étudié, cette énergie est répartie assez uniformément. Partout à l'extérieur du port, la valeur de $E^{*}$ est très voisine de 4 , ce qui est exactement la somme de l'énergie de la houle incidente plus l'énergie d'une houle réfléchie d'égale magnitude à cette première. A l'intérieur du bassin, $\mathrm{E}^{*}$ varie entre 4 , sur son axe de symétrie, et 9 , dans quelques points localisés principalement sur la frontière parallèle à l'axe de symétrie du bassin.

L'énergie totale adimensionnelle est représentée sur la figure 5 par son iso-valeur au carré ; ceci afin de mieux cerner les amplitudes relatives maximales possibles, car dans les ventres d'oscillation celles-ci valent $\mathrm{Ep}^{*^{1 / 2}}$.

\subsection{Distribution des phases}

La figure 6 montre les distributions des phases $0^{\circ}, 90^{\circ}, 180^{\circ}$ et $270^{\circ}$, dans un bassin avec. des frontières parfaitement réfléchissantes. On constate qu'à l'intérieur du bassin les 4 phases représentées se concentrent aux environs des noeuds d'oscillation. On observe également que des traits discontinus matérialisent les phases, mais si l'on met ensemble les points de phases opposées $\left(0^{\circ}-180^{\circ}, 90^{\circ}-270^{\circ}\right)$ on obtient une ligne continue. Enfin, la présence d'un nombre important de points amphidromiques (amplitude nulle et phase indéfinie) est clairement visible. La distance qui sépare deux points amphidromiques voisins est très proche d'une demilongueur d'onde. 


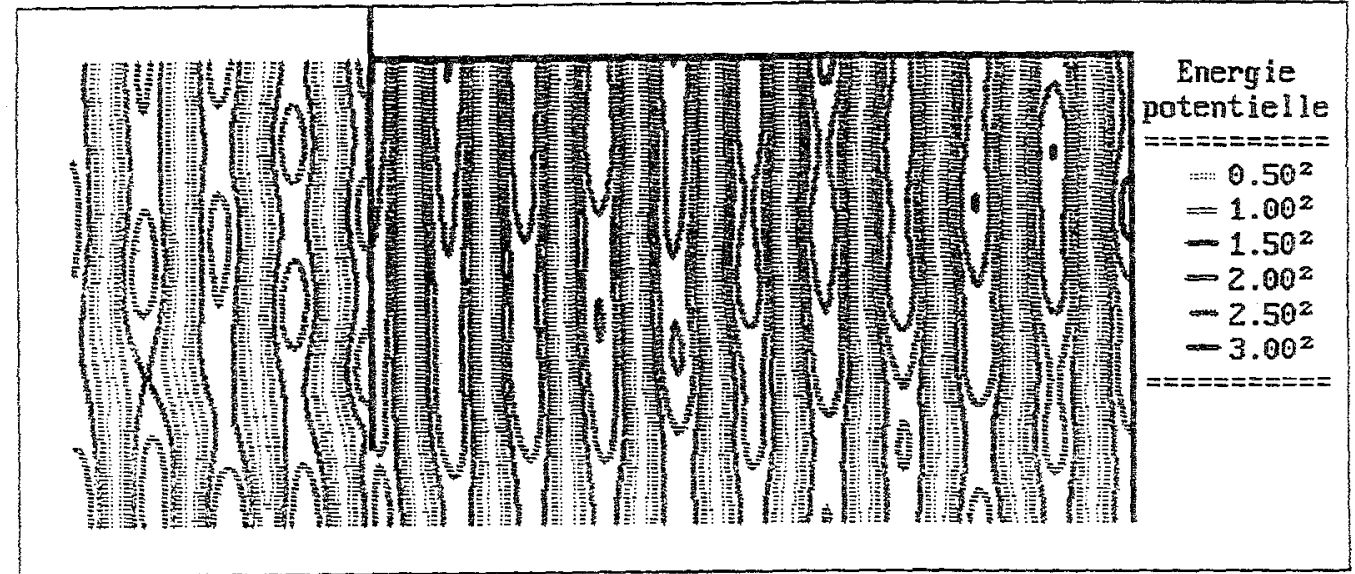

Fig.3.- Distribution de l'énergie potentielle adimensionnelle Ep* dans un bassin entouré de frontières parfaitement réfléchissantes.

L'amplitude locale de la houle sur l'amplitude de la houle incidente vaut $\mathrm{Ep}^{*^{1 / 2}}$.

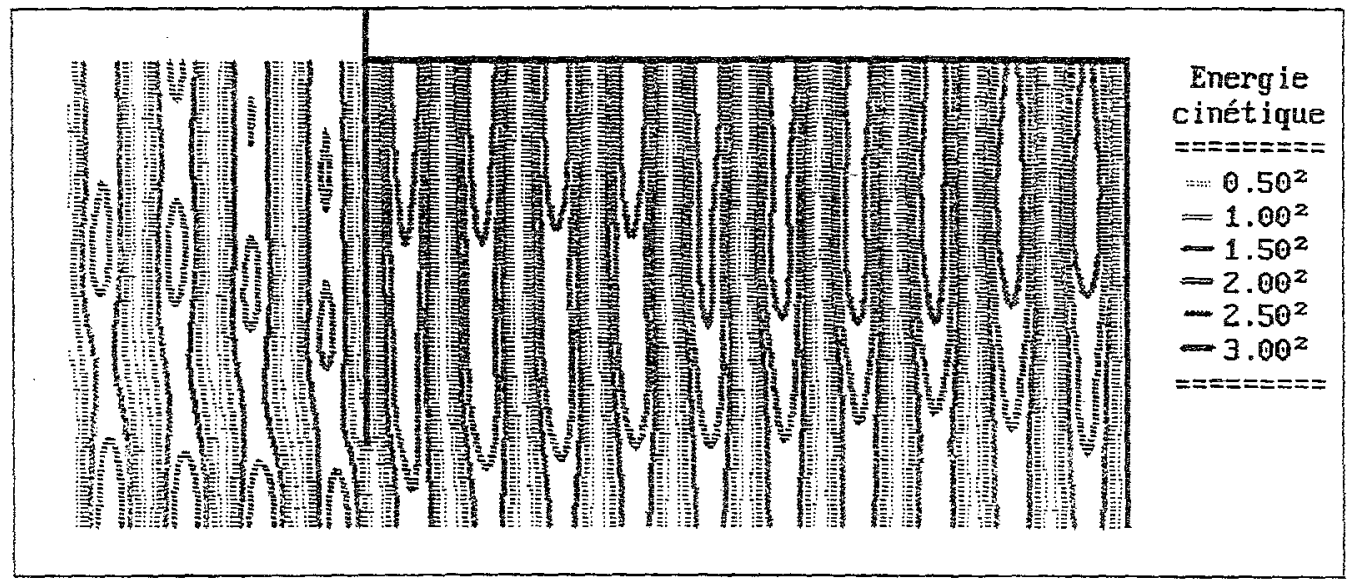

Fig.4.- Distribution de l'énergie cinétique adimensicnnelle Ec* dans un bassin entouré de frontières parfaitement réfléchissantes, en faibles profondeurs.

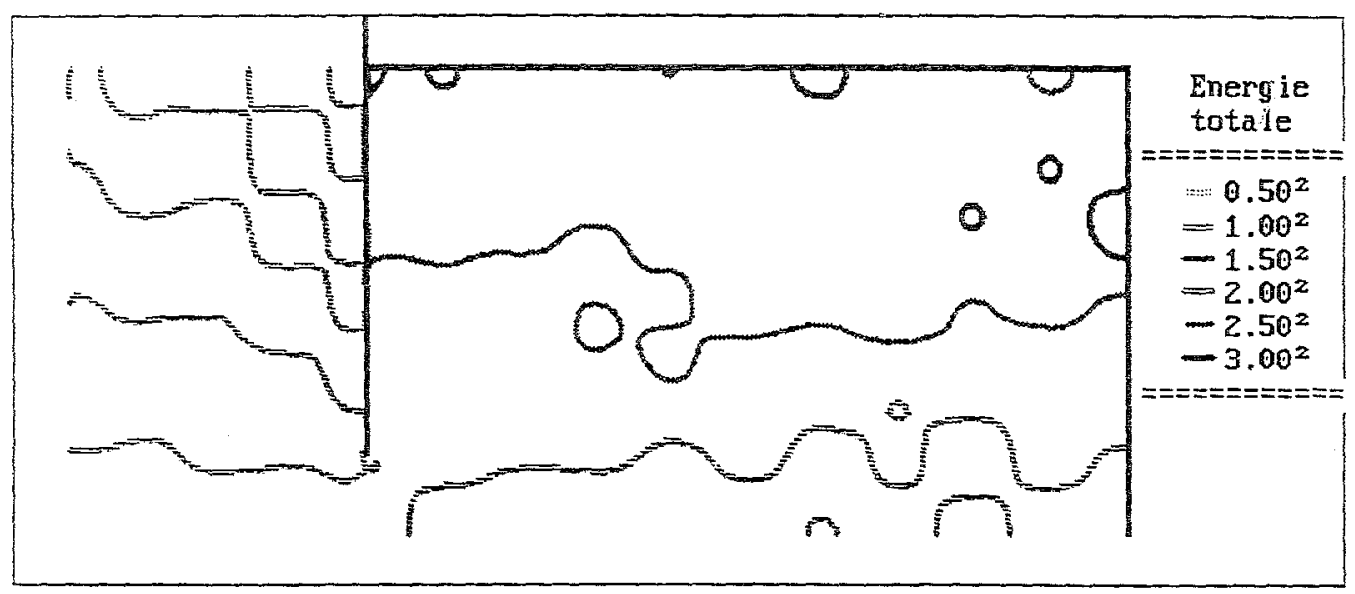

Fig.5.- Distribution de l'énergie totale adimensionnelle $E^{*}$ dans un bassin entouré de frontières parfaitement réfléchissantes, en faibles profondeurs. 


\subsection{Distribution des énergies transmises}

La figure 7 montre la distribution de l'énergie transmise adimensionnelle Et* dans un bassin avec des frontières parfaitement réfléchissantes. La première observation d'importance est que l'énergie transmise vers les parois est nulle ; ceci étant dû au fait que les parois sont incapables d'absorber de l'énergie. La deuxième observation est que le débit net d'énergie à travers l'accès est nul (l'énergie transmise vers l'intérieur du port sur les côtés de l'accès est compensée par une énergie transmise vers l'extérieur, laquelle se produit sur la partie centrale de l'accès); ceci montre que le modèle reproduit une dissipation nulle d'énergie à l'intérieur du bassin. La troisième observation est que l'énergie circule autour des points amphidromiques et que les sens de la circulation sont inversés entre deux tourbillons d'énergie voisins ; les tourbillons d'énergie transmise forment des carrés ; chaque côté des carrés est égal à une demi-longueur d'onde.

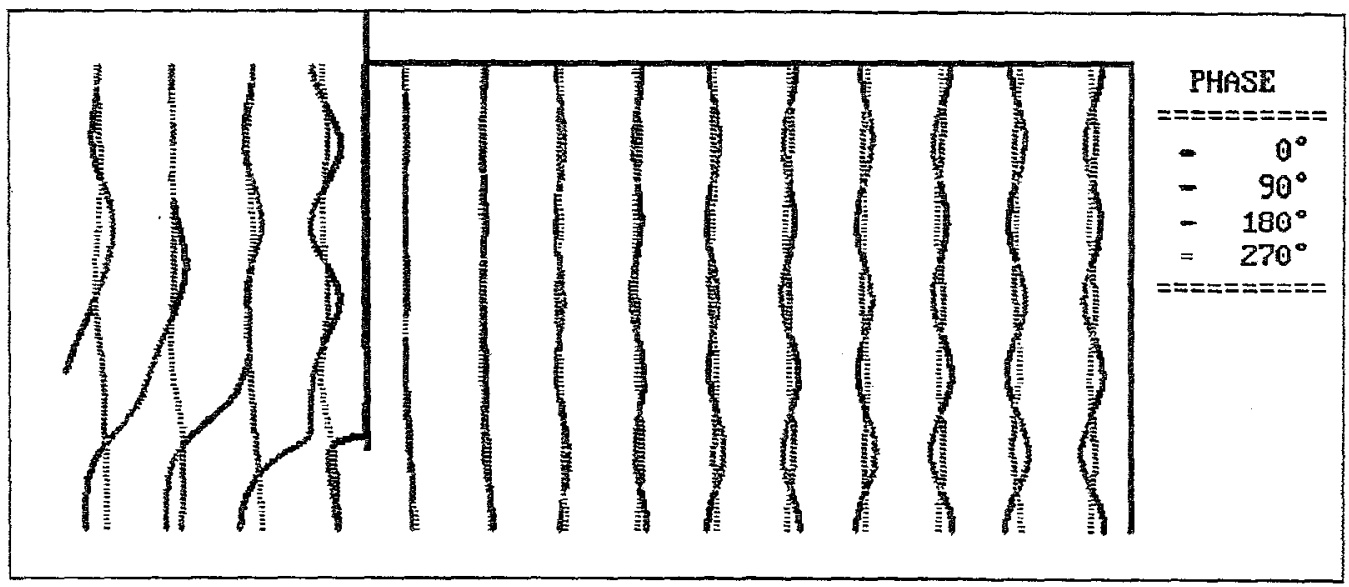

Fig.6.- Distribution des phases dans un bassin entouré de frontières parfaitement réfléchissantes.

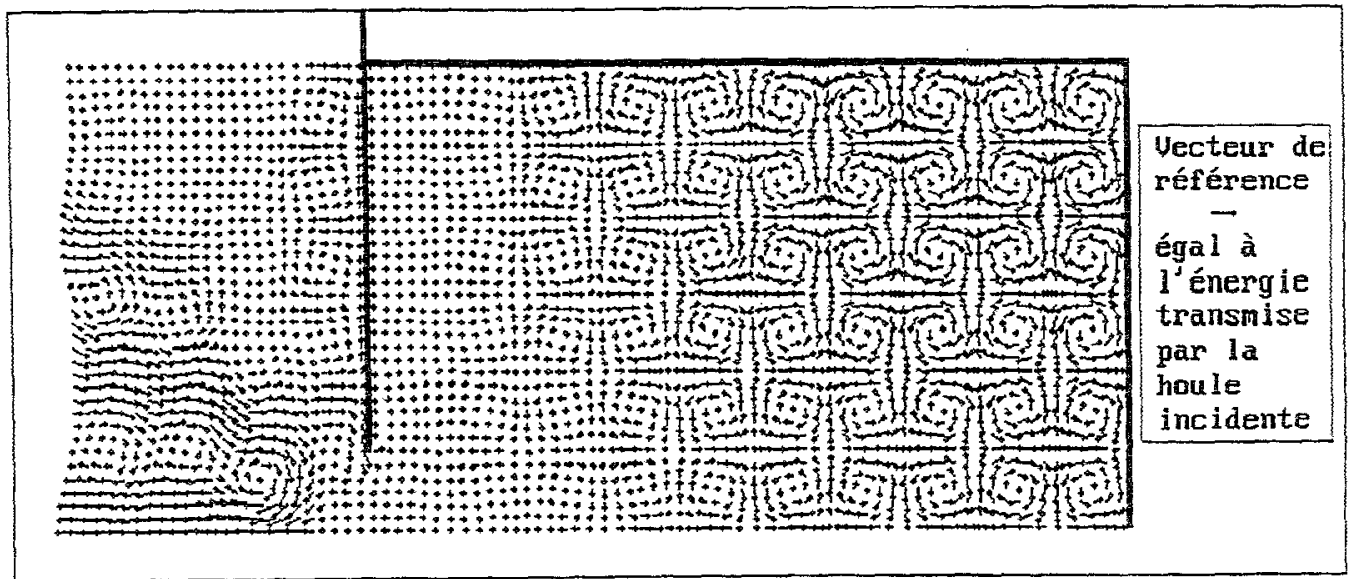

Fig.7.- Distribution de l'énergie transmise adimensionnelle dans un bassin entouré de frontières parfaitement réfléchissantes. 


\section{Conclusions}

La méthodologie numérique suivie dans cette étude pour simuler une réflexion nulle ou partielle au niveau des parois latérales d'un bassin permet létude systématique de la propagation d'une houle monochromatique dans un bassin de forme quelconque avec un degré d'approximation tout à fait satisfaisant d'un point de vue pratique.

La démarche permettant de tenir compte d'une réflexion nulle ou partielle comporte une première itération de calcul numérique où les conditions aux limites sont calculées grâce aux équations $(7)$ et $(8)$ en prenant $y=0^{\circ}$. Pour la deuxième itération et pour les suivantes, les conditions aux limites sont calculées grâce à l'équation (11) en utilisant les phases estimées lors de l'itération précédente.

L'équation (11), propre à cette étude, permet de reproduire soit un clapotis partiel issu d'une houle progressive cylindrique abordant une paroi lorsqu'elle est partiellement réfléchissante soit une réflexion nulle lorsque cette paroi est complètement absorbante.

Dans cette étude on présente les distributions des énergies potentielle, cinétique et totale, dans un bassin en eaux peu profondes entouré de parois parfaitement réfléchissantes ( ces représentations sont également possibles pour chaque valeur de $\mathrm{kd}$ ). On constate dans ce cas une résonance qui se manifeste par une forte variation dans l'espace des énergies potentielle et cinétique avec un grand nombre des maximums et minimurns. En revanche, la distribution de l'énergie totale est assez uniforme et permet une identification simple des secteurs les plus exposés à l'action de la houle dans un port.

La représentation des phases permet de localiser les éventuels points amphidromiques autour desquels l'énergie circule. Enfin, la représentation de l'énergie transmise permet une bonne compréhension de la circulation d'énergie engendrée par une houle progressive incidente. L'évaluation de l'énergie absorbée par chaque partie des parois d'un port est égailement possible grâce à cette demière représentation.

\section{Références bibliographiques}

[1] Berkhoff, J.C.W., "Mathematical models for simple harmonic water wave diffraction and refraction", Delft Hydraulics Lab., Rep. 168, 1976.

[2] Isaacson, M. and S. Qu, "Waves in a harbour with partially reflecting boundaries", Coastal Engineering, Elsevier, Vol. 14, n³ 1990, pp 193-214.

[3] Pos, J.D. and F.A. Kilner, "Breakwater gap wave diffraction : an experimental and numerical study", ASCE, J. Waterw. Harbours, Coastal Ocean Eng., 113 (1), 1987, pp 1-21.

[4] Shore Protection Manual, Coastal Engineering Research Center, U.S. Army Corps of Engineers, Viksburg, Miss., 1984. 